\title{
COMPOSICIÓN NUTRICIONAL DE LA ALGA ROJA Rodhymenia howeana DE LA BAHÍA DE ANCÓN, PERÚ
}

\author{
Naddia Carolina RojasVega a, Rubén Valdivieso Izquierdo ${ }^{\text {, }}$ Inés Arnao Salas ${ }^{\mathrm{b}}$
}

\begin{abstract}
RESUMEN
El presente estudio tuvo como objetivo evaluar la composición nutricional del alga roja Rodhymenia howeana. Ensayos realizados: análisis proximal determinado según la AOAC, contenido de minerales por espectroscopia de absorción atómica, el cómputo aminoacídico mediante cromatografía de intercambio catiónico usando un analizador de aminoácidos, el contenido de vitamina $\mathrm{C}$ y fenoles totales por espectrofotometría. Los resultados más resaltantes del análisis proximal fueron $28,56 \%$ de proteína; $21,08 \%$ de cenizas y trazas de extracto etéreo (lípidos) en muestra seca. Del contenido de minerales: hierro $502 \mathrm{mg} / \mathrm{kg}$ y zinc $18,54 \mathrm{mg} / \mathrm{kg}$ de muestra seca de alga. Los aminoácidos esenciales más abundantes $(\mathrm{mg} / 100$ $\mathrm{g}$ de muestra seca) fueron: valina 95, leucina 116 y lisina 116,2 y entre los no esenciales resaltaron: alanina 139, ácido aspártico 244 y ácido glutámico 313. De los componentes antioxidantes el contenido de vitamina $\mathrm{C}$ fue de $1,12 \mathrm{mg}$ en $100 \mathrm{~g}$ de muestra seca y de fenoles totales fue de 980,2 mg EAG /100 g de muestra seca. Conclusiones: el alga roja Rodhymenia howeana es un alimento nutritivo y contiene compuestos antioxidantes.
\end{abstract}

Palabras clave: composición nutricional, Rodhymenials, algas rojas, minerales, aminoácidos.

\section{NUTRITIONAL COMPOSITION OF RED SEAWEED Rodhymenia howeana FROM ANCON BAY, PERU}

\begin{abstract}
The objective of this study was to evaluate the nutritional composition of the red alga Rodhymenia howeana. Tests performed: proximal analysis determined according to AOAC, mineral content by atomic absorption spectroscopy, amino acid count by cation exchange chromatography using an amino acid analyzer, vitamin $\mathrm{C}$ content and total phenols by spectrophotometry. The most important results of the proximal analysis were $28.56 \%$ of protein; $21.08 \%$ ash and traces of ethereal extract (lipids) in dry sample. Of the mineral content: iron $502 \mathrm{mg} / \mathrm{kg}$ and zinc $18.54 \mathrm{mg} / \mathrm{kg}$ of dry sample of algae. The most abundant essential amino acids (mg / $100 \mathrm{~g}$ of dry sample) were: valine 95, leucine 116 and lysine

\footnotetext{
${ }^{a}$ Nutricionista. Unidad de Posgrado de la Facultad de Medicina. Universidad Mayor de San Marcos.

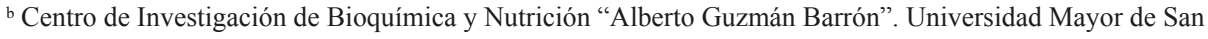
Marcos.
} 
116.2 and among the non-essential ones relevant: alanine 139, aspartic acid 244 and glutamic acid 313. Of the antioxidant components the content of vitamin $\mathrm{C}$ was $1.12 \mathrm{mg}$ in $100 \mathrm{~g}$ of dry sample and of total phenols was $980.2 \mathrm{mg}$ EAG / $100 \mathrm{~g}$ of dry sample. Conclusions: the red seaweed Rodhymenia howeana is a nutritious food and contains antioxidant compounds.

Key words: Nutrition facts, Rodhymenials, red seaweeds, minerals, amino acids

\section{INTRODUCCIÓN}

Las algas marinas han sido utilizadas como alimento en humanos, y en menor proporción como fertilizantes y aditivos para alimentos de animales. Las algas como alimento tienen un alto consumo en países asiáticos como China, Japón y Corea, y su demanda ha aumentado en países de Norte América, Sudamérica y Europa, principalmente por la gran migración de asiáticos a otros países del mundo ${ }^{1}$. Estos alimentos son importantes reservorios de nutrientes, pues presentan un bajo contenido de calorías y lípidos lo cual beneficiarán a las personas con patologías como sobrepeso, obesidad, diabetes y dislipidemias. Su elevada concentración de minerales, vitaminas antioxidantes y moléculas bioactivas como polifenoles con amplias potencialidades terapéuticas ayudarán a la prevención de enfermedades relacionadas con estrés oxidativo ${ }^{2},{ }^{3}$.

Nuestro país posee vastos recursos ya que existen 228 especies de macroalgas de las cuales 160 son rojas, 31 pardas y 37 verdes. Las especies de macroalgas marinas con importancia económica son Chondracanthus chamissoi, Gracilariopsis lemanaeformis, Porphyra spp, Lessonia nigrescens, Lessonia trabeculata, Macrocystis integrifolia, Macrocystis pyrifera y Ulva $\mathrm{spp}^{1}$. En tanto no se desarrollen investigaciones en este campo, no serán tomadas en cuenta las más de 100 especies de macroalgas en el mar peruano y la Antártica.

Dentro de este grupo de macroalgas no estudiadas está la especie Rodhymenia howeana que en el pasado fue empleada como parte de la alimentación de la población local. Es una especie distribuida en costas de Perú y Chile. En nuestro país se encuentra en: Bahía de Chimbote, Bahía de Ancón, Islas Chincha y La Punta, Callao. Existe poca investigación en el país sobre las algas marinas en general, a pesar de ser un recurso abundante, económico y con muchos potenciales; por este motivo se considera prioritario conocer su composición nutricional y capacidad antioxidante para revalorizar este alimento marino ya que beneficiará a nuestra población en general.

El objetivo del presente estudio es determinar la composición nutricional mediante el análisis proximal, contenido de aminoácidos, mineral, vitamina $\mathrm{C}$ y de fenoles totales del alga Rodhymenia howeana. 


\section{PARTE EXPERIMENTAL}

\section{Muestra y colecta de la materia prima}

Diseño: enfoque cuantitativo, descriptivo, observacional y transversal. Se recolectaron 10 $\mathrm{kg}$ de alga marina roja Rodhymenia howeana, durante el periodo de octubre - noviembre 2017 en la Bahía de Ancón en la ciudad de Lima, Perú (1145.9 S $77^{\circ} 11.7$ W). Las algas seleccionadas fueron lavadas para remover la materia extraña y enjuagada repetidamente con agua destilada. Las algas frescas fueron colocadas en un conservador portátil de alimentos con hielo seco. Los especímenes fueron identificados y autenticados en el Museo de Historia Natural de la Universidad Mayor de San Marcos.

Los reactivos: ácido ascórbico y DPPH fueron de la casa Sigma-Aldrich y alcohol etílico, Folin Ciocalteu, ácido gálico, carbonato de sodio y ácido tricloroacético de la casa Merck.

\section{Composición proximal}

El análisis proximal se realizó en el Laboratorio de Bioquímica, Nutrición y Alimentación Animal de la Facultad de Veterinaria de la Universidad Nacional Mayor de San Marcos, donde emplearon la metodología analítica de AOAC 930.15 para humedad, AOAC 984.13 para proteína cruda, ISO 6865 AOAC 978.10 para fibra cruda, AOAC 942.05 para cenizas y AOAC 2003.05 para grasa. El extracto no nitrogenado se obtuvo por diferencia de los componentes ${ }^{4}$. Los resultados se expresan en base húmeda $\%$ y base seca $\%$.

\section{Composición mineral}

Las determinaciones mediante el método espectroscopia de absorción atómica (EAA) de los minerales manganeso, zinc, hierro, magnesio, cobre y selenio obtenidos se realizó en la Unidad de Servicios de Análisis Químicos de la Facultad de Química e Ingeniería Química de la Universidad Nacional Mayor de San Marcos. Los resultados fueron expresados en mg/ $\mathrm{kg}$ de muestra seca.

\section{Composición aminoacídico}

El análisis aminoacídico se realizó en el Instituto de Investigación de Ciencias de la Alimentación (CIAL) en Madrid y la metodología usó una columna cromatográfica de intercambio catiónico en un analizador de aminoácidos Biochrom serie 30 (Biochrom, Cambridge, EE.UU.) Las muestras fueron previamente hidrolizadas con $\mathrm{HCl} 6 \mathrm{~N}$ durante 21 horas a $110^{\circ} \mathrm{C}$ y los aminoácidos fueron derivatizados con o-ftalaldehído (OPA) en una precolumna. La detección fue realizada por absorbancia a $440 \mathrm{~nm}$. El contenido de aminoácidos se expresa en $\mathrm{mg} / \mathrm{g}$ de proteína \pm desviación estándar.

\section{Contenido de vitamina c}

La metodología empleada fue la descrita por Jagota y Dani ${ }^{5}$. Para este ensayo se añadió $120 \mu \mathrm{l}$ de ácido tricloroacético (TCA) al $10 \%$ al extracto acuoso y luego fue centrifugado. Posteriormente, se utilizó $600 \mu \mathrm{l}$ de reactivo de Folin-Ciocalteu al $10 \%$ con $480 \mu \mathrm{l}$ del sobrenadante. Se realizó la lectura a $760 \mathrm{~nm}$ en el espectrofotómetro (S-20, Marca Boeco) posterior a su agitación y reposo de 10 minutos. Los resultados se expresaron en mg de ácido ascórbico en $100 \mathrm{~g}$ de muestra seca. 


\section{Contenido de fenoles totales}

El ensayo se realizó según Singleton, Orthofer y Lamuela-Raventos ${ }^{6}$. Para este ensayo se colocó $1250 \mu \mathrm{l}$ de reactivo de Folin-Ciocalteu, $500 \mu \mathrm{l}$ del extracto acuoso, $1000 \mu \mathrm{l}$ de solución de $\mathrm{Na}_{2} \mathrm{CO}_{3}$ al 7,5\% w/v. Después de 45 minutos de incubación en baño de María a $50^{\circ} \mathrm{C}$ se leyó a $760 \mathrm{~nm}$ en el espectrofotómetro (S-20, Marca Boeco). Los resultados fueron expresados en equivalentes de ácido gálico en $100 \mathrm{~g}$ de alga seca. La concentración de polifenoles fue derivada de una curva de ácido gálico en el rango de 10 a $50 \mu \mathrm{g} / \mathrm{mL}$.

\section{Análisis estadístico}

Todos los datos fueron analizados en tres experimentos independientes y se utiliza la estadística descriptiva presentando los resultados como promedio y desviación estándar.

\section{RESULTADOS Y DISCUSIÓN}

Los carbohidratos, proteínas, lípidos y vitaminas se proporcionan al cuerpo humano a través de diferentes fuentes de alimentos entre los cuales deben ser considerados las algas marinas, abundantes en nuestras costas peruanas.

El análisis proximal nos permite conocer el contenido de estos nutrientes, y los resultados para el alga Rodhymenia howeana se presentan en la tabla 1.

Tabla 1. Composición proximal del alga Rodhymenia howeana

\begin{tabular}{lcc}
\hline Componente & Base húmeda $\%$ & $\begin{array}{l}\text { Base } \\
\%\end{array}$ \\
\hline Humedad & 79,03 & 20,77 \\
\hline Proteína & 5,99 & 28,56 \\
\hline Extracto etéreo & trazas & trazas \\
\hline Fibra cruda & 2,32 & 11,04 \\
\hline Cenizas & 4,42 & 21,08 \\
\hline $\begin{array}{l}\text { Extracto } \\
\text { nitrogenado }\end{array}$ & 8,24 & 39,32 \\
\hline
\end{tabular}

Tal como en las plantas terrestres, las algas marinas también son una fuente rica de elementos nutricionales, pues presentan altos niveles de fibra, minerales, ácidos grasos omega 3 y concentraciones moderadas de lípidos y proteínas, disponibles en la mayoría de las algas comestibles, son aportes que deben ser considerados como una importante fuente de alimentos para la nutrición humana. 
Un componente valorado en todo alimento es su contenido proteico, tal es así que para la especie Rodhymenia howeana su contenido proteico fue de $28,56 \%$ en base seca. En comparación con otros autores, los valores más bajos (7,8 \% y 8,9\% en muestra seca) son presentados por Xiren y Aminah ${ }^{7}$ en especies rodofita, Kappaphycus alvarezii de Malasia. Este resultado es diferente a lo reportado por Frikha ${ }^{3}$ en algas tunecinas, donde el mayor contenido de proteínas fue de $14 \%$ en muestra seca para el alga roja Ceramium diaphanum, valor que coincide con el de Nehal Osman et al para el alga verde Caulerpa serrulata. Otros autores como Alvarez y Sierra ${ }^{9}$ obtienen valores de $20 \%$ de contenido de proteínas en muestra seca para la especie roja Gracilariopsis tenuifrons, los que se asemejan a las especies brasileras Hypnea musciformis y Solieria filiformis, ambas rodofitas, con 17 \% y 20 $\%$, respectivamente. Sin embargo, la especie coreana Porphyra tenera y Porphyra haitanesis con $32 \%$ y $36 \%$, reportada por Hwang et $a l^{10}$, es la que supera en contenido de proteína cruda a todas las anteriores. Debemos señalar que los países asiáticos emplean algas que se cultivan de manera comercial para mejorar su calidad, lo que les permite concentrar todos sus componentes, de allí los elevados valores en proteínas obtenidos.

La cantidad de lípido, respecto a otras algas rojas, se mantiene con un valor similar, siempre menores a $3 \%$, como en el reino vegetal y en relación al porcentaje de fibra este es menor que otras especies. Las diferencias en la composición proximal son atribuibles a factores como el clima, la temperatura, el $\mathrm{pH}$, diferencias geográficas, la especie y la estacionalidad.

El contenido mineral de manganeso, magnesio, zinc y hierro se presentan en la tabla 2, siendo el elemento más destacable el hierro; no fueron detectados, selenio ni cobre teniendo en consideración el límite de detección para estos elementos según la técnica empleada de 10 $\mathrm{mg} / \mathrm{kg}$ y $0,5 \mathrm{mg} / \mathrm{kg}$, respectivamente.

Tabla 2. Contenido mineral del alga Rodhymenia howeana

\begin{tabular}{cc}
\hline Mineral & $\begin{array}{c}\mathrm{mg} / \mathrm{kg} \text { de muestra } \\
\text { seca }\end{array}$ \\
\hline Cobre & No Detectable \\
\hline Selenio & No Detectable \\
\hline Manganeso & 7,1 \\
\hline Zinc & 11,8 \\
\hline Hierro & 502 \\
\hline Magnesio & 1,8 \\
\hline
\end{tabular}


Es indispensable la incorporación al organismo de ciertos oligoelementos ya que forman parte del núcleo activo de las ciertas macromoléculas, algunos funcionan como cofactores de enzimas con capacidad antioxidante como la superóxido dismutasa, que contiene zinc, cobre y manganeso.

El elemento traza más destacable de los analizados en R. howeana, es el hierro y Osman et $a l^{8}$, quienes evaluaron la especie clorofita Caulerpa serrulata, obtuvieron una concentración de hierro de $70 \mathrm{mg} / \mathrm{kg}$. En las especies Kappaphycus alvarezii y Gracilariopsis tenuifrons, ambas algas rojas evaluadas por Alvarez ${ }^{9}$, el contenido de hierro también es menor, con 56,27 y 204,83 y mg/kg, respectivamente, al igual que en Porphyra spp, Chondrus crispus, Palmaria palmata y Laminaria spp con concentraciones medias de hierro de $106 \mathrm{mg} / \mathrm{kg}$ según reporte de Ruíz et al ${ }^{11}$. De todos los estudios considerados en el presente artículo, la especie Porphyra haitanensis, estudiada por Hwang ${ }^{10}$, presentó valores de hierro de $700 \mathrm{mg} / \mathrm{kg}$, que son mayores a los de la alga roja peruana.

Más del $40 \%$ de los niños y niñas menores de 5 años y cerca del $21 \%$ en mujeres de edad fértil son diagnosticados de anemia en nuestro país, según el Plan Nacional de Reducción y Control de la Anemia del año 2017. El hierro es un nutriente esencial para el ser humano ya que participa en el transporte de oxígeno en la sangre y lo almacena en el músculo. Las necesidades de hierro diarias varían con la edad y son más importantes en niños, mujeres en edad fértil y durante el embarazo ${ }^{12}$. Siendo este mineral el más abundante de nuestra alga estudiada, podría ser empleado como suplemento útil en la prevención y/o tratamiento de la anemia.

El zinc es un mineral extraordinariamente versátil que forma parte de más de 100 enzimas, está relacionado con el crecimiento y desarrollo durante la pubertad y la adolescencia, la actividad de la vitamina A, la síntesis de enzimas pancreáticos, es esencial para mantener el sentido del gusto y, por tanto, el apetito, para facilitar la cicatrización de las heridas y para el normal desarrollo del feto, entre otras importantes funciones ${ }^{12},{ }^{13}$.

Este mineral fue evaluado por Osman et $a l^{8}$ en la especie clorofita Caulerpa serrulata con resultados de $13,1 \mathrm{mg} / \mathrm{kg}$ de zinc y en la especie coreana Porphyra columbina, con valores similares a lo encontrado en el presente estudio. Para las algas rojas Gracilariopsis tenuifrons y Kappaphycus alvarezii, evaluadas por Álvarez y Sierra ${ }^{9}$, con un contenido de zinc fue 16,32 y $19,40 \mathrm{mg} / \mathrm{kg}$, respectivamente, que son mayores a lo encontrado en nuestra alga peruana.

El contenido mineral en algas es mayor que el de las plantas terrestres, por lo tanto, las algas marinas pueden ser una fuente importante de minerales porque algunos de estos elementos trazas, están ausentes en hortalizas de tierra o sólo se encuentran en concentraciones muy pequeñas.

Un factor determinante en la calidad de las fuentes proteicas es la composición en aminoácidos, tal como se presenta en la tabla 3 para nuestra alga en estudio, en la cual se observa la presencia de 19 aminoácidos diferentes expresados en $\mathrm{mg} / \mathrm{g}$ de proteína. El triptófano no fue determinado debido a que se destruye por hidrólisis ácida. 
Tabla 3. Composición aminoacídico del alga Rodhymenia howeana

$(\mathrm{mg} / \mathrm{g}$ proteína en muestra seca)

\begin{tabular}{lc}
\hline \multicolumn{1}{c}{ Aminoácido } & Aminoácido \\
\hline Ácido aspártico + Asparagina & $8,55 \pm 0,27$ \\
\hline Treonina & $3,30 \pm 0,16$ \\
\hline Serina & $4,10 \pm 0,13$ \\
\hline Ácido glutámico + Glutamina & $10,97 \pm 0,47$ \\
\hline Glicina & $3,14 \pm 0,11$ \\
\hline Alanina & $4,90 \pm 0,17$ \\
\hline Cisteína & $1,74 \pm 0,01$ \\
\hline Valina & $3,32 \pm 0,09$ \\
\hline Metionina & $2,38 \pm 0,20$ \\
\hline Isoleucina & $2,58 \pm 0,18$ \\
\hline Leucina & $4,07 \pm 0,29$ \\
\hline Tirosina & $1,86 \pm 0,05$ \\
\hline Fenilalanina & $3,13 \pm 0,12$ \\
\hline Histidina & $1,02 \pm 0,04$ \\
\hline Lisina & $4,06 \pm 0,15$ \\
\hline Arginina & $2,81 \pm 0,13$ \\
\hline Prolina & $3,00 \pm 0,18$ \\
\hline
\end{tabular}

Una mejor apreciación de este contenido es clasificarlos en aminoácidos esenciales y no esenciales, lo cual nos va a facilitar la discusión de los resultados, tal como se observa en la tabla 4, en donde los valores se expresan en mg/ $100 \mathrm{~g}$ de muestra seca.

Tabla 4. Contenido de aminoácidos esenciales y no esenciales del alga Rodhymenia howeana en muestra seca

\begin{tabular}{lcr|lrr}
\hline $\begin{array}{l}\text { Aminoácidos } \\
\text { esenciales }\end{array}$ & $\mathrm{mg} / 100 \mathrm{~g}$ & $\%$ & $\begin{array}{l}\text { Aminoácidos no } \\
\text { esenciales }\end{array}$ & $\mathrm{mg} / 100 \mathrm{~g}$ & $\%$ \\
\hline Histidina & 29,1 & 1,6 & $\begin{array}{l}\text { Ácido aspártico }+ \\
\text { asparagina }\end{array}$ & 244,2 & 13,2 \\
\hline Treonina & 94,2 & 5,1 & $\begin{array}{l}\text { Ácido glutámico + } \\
\text { lutamina }\end{array}$ & 313,3 & 16,9 \\
\hline Arginina & 80,3 & 4,3 & Serina & 117,1 & 6,3 \\
\hline Lisina & 116 & 6,3 & Glicina & 89,7 & 4,8 \\
\hline Metionina & 68 & 3,7 & Prolina & 85,7 & 4,6 \\
\hline Fenilalanina & 89,4 & 4,8 & Tirosina & 53,1 & 2,9 \\
\hline Valina & 94,8 & 5,1 & Cisteina & 49,7 & 2,7 \\
\hline Isoleucina & 73,7 & 4,0 & Alanina & 139,9 & 7,5 \\
\hline Leucina & 116,2 & 6,3 & & & \\
\hline Total AAE & 761,7 & 41,1 & Total AANE & 1092,7 & 58,9 \\
\hline
\end{tabular}

Total: $1854,4 \mathrm{mg}$ 
De los aminoácidos esenciales destacan la treonina y valina con 5,1\% y lisina y leucina con $6,3 \%$, siendo la histidina la que presentó el valor más bajo. En comparación con la investigación de Xiren y Aminah ${ }^{7}$ quienes emplean la alga roja Kappaphycus alvarezii de Lawakii (Malasia) los aminoácidos resaltantes fueron leucina con 0,32 mg/100 g, valina y fenilalanina con resultados de $0,24 \mathrm{mg} / 100 \mathrm{~g}$ y en caso de la misma especie pero de la localidad de Sabah destaca la leucina, siendo el valor más elevado de 0,56 mg/100 g de muestra seca. Estos aminoácidos esenciales tienen valores menores al ser comparados con los de nuestro estudio, donde el contenido de leucina y de valina fue de 116,2 y de 94,8 $\mathrm{mg}$ en $100 \mathrm{~g}$ de muestra seca. Con respecto a lo reportado por Hwang et a $\mathrm{l}^{10}$ que evaluaron la especie coreana Porphyra tenera y la especie china Porphyra haitanensis destacan isoleucina con $46,67 \mathrm{mg} / 100 \mathrm{~g}$ y valina con $33,48 \mathrm{mg} / 100 \mathrm{~g}$ de muestra seca y en nuestro estudio dichas concentraciones son mayores (74 y $94 \mathrm{mg} / 100 \mathrm{~g}$ de muestra seca de los aminoácidos ya mencionados), mientras que en la especie china $P$. haitanensis predomina el contenido de treonina con $86,43 \mathrm{mg} / 100 \mathrm{~g}$ que es similar con lo encontrado en el alga peruana (94 mg/100 g), ambas en base seca. Sin embargo, las diferencias son notables con el alga roja chilena Chondracantus chamissoi investigada por Ortiz ${ }^{2}$ ya que sus resultados para histidina, leucina y lisina, con valores de 879, 706 y $593 \mathrm{mg} / 100 \mathrm{~g}$ de muestra seca, respectivamente, son significativamente más elevados que en $R$. howeana. Sin embargo, la concentración de aminoácidos ramificados (leucina, valina e isoleucina) en nuestro estudio es el doble que lo analizado en la especie coreana $P$. tenera, que tiene un contenido de 108 $\mathrm{mg}$ de los aminoácidos mencionados. Si tomamos en cuenta los valores porcentuales de los aminoácidos ramificados observamos que los porcentajes de isoleucina para la especie $R$. howeana es de $4 \%$, C. chamissoi de 4,5\% y K. alvarezzi de Lawakii con 5,5\% que son proporcionalmente similares. Algo parecido ocurre con el aminoácido valina donde los valores son de $5 \%, 6 \%$ y $6,3 \%$ en el mismo orden de las tres especies mencionadas. No obstante, con leucina las concentraciones en las especies C. chamissoi y K. alvarezzi son de $8 \%$ y $9,6 \%$ ambos resultados son mayores a los presentados en el alga de estudio con $6 \%$.

La especie Rodhymenia howeana es rica en ácido glutámico (16,9\%), ácido aspártico (13\%), alanina $(7,5 \%)$ y serina $(6,3 \%)$. Realizando una comparación de los aminoácidos no esenciales con otras especies observamos que el ácido glutámico varía del $12 \%$, al $14 \%$ y el aspártico del $10 \%$ al 13,8 \% en las especies $K$. alvarezii, y $C$. chamissoi, siendo estos aminoácidos ácidos los más abundantes, al igual que en nuestro caso.

Debemos destacar el contenido de cisteína en $R$. howeana, ya que es precursor en la síntesis de glutatión, un potente antioxidante de nuestro organismo. Este aminoácido no ha sido determinado en las especies estudiadas por Xiren y Aminah ${ }^{7}$ y su valor es mínimo en C. chamissoi.

Debido a las diferentes metodologías empleadas en la determinación del contenido de aminoácidos, las unidades en que se expresan los resultados, la composición de los hidrolizados, entre otros, no siempre resulta fácil una comparación. Si expresamos estos datos en porcentaje, encontramos que cien gramos de $R$. howeana aporta 761,7 $\mathrm{mg}$ de aminoácidos esenciales, lo que representa el $41 \%$, esto es equivalente a una relación de aminoácidos esenciales (AAE) y 
no esenciales (AANE) de 0,71. Este valor es muy cercano a los de $K$. alvarezzi obtenidos de dos localidades de Malasia con valores de 0,74 y 0,79 pero diferente a Ch. chamissoi (Chile) en donde esta relación alcanza 1,47.

Finalmente, podemos expresar que existe una gran variabilidad en el contenido de los aminoácidos de las muestras de alga ya que es conocida que esta composición es altamente influenciada por la localización geográfica y las condiciones ambientales locales, además de la sensibilidad de la técnica empleada en la cuantificación de los aminoácidos.

El ácido ascórbico es un agente antioxidante y reductor poderoso y puede, por lo tanto, reducir la acción perjudicial de los radicales libres, sus principales funciones son neutralizar el oxígeno singlete $(\mathrm{O} 2)$, capturar radicales hidroxilos, aniones superóxidos y regenerar la forma oxidada de la vitamina E. Es también importante para mejorar la absorción del hierro no-hemínico en alimentos de origen vegetal. Es necesario para la formación y mantenimiento adecuado del material intercelular, sobre todo del colágeno. No se sintetiza en el organismo, por lo que debe ser aportada por la dieta ${ }^{12},{ }^{13}$.

El contenido de vitamina $\mathrm{C}$ del alga roja Rhodymenia howeana fue de 1,12 $\pm 0,04 \mathrm{mg}$ en $100 \mathrm{~g}$ de muestra seca. El valor más bajo lo presentó la especie rodofita Corallina officinalis evaluada por Ismail ${ }^{14}$ con resultados de $0,67 \mathrm{mg} / 100 \mathrm{~g}$. El valor más elevado fue de 21,6 $\mathrm{mg} / 100 \mathrm{~g}$ de muestra seca reportado por Yildiz et al ${ }^{15}$ en la especie roja Gracilaria bursapastoris. Estos resultados de vitamina $\mathrm{C}$, tan variados, podrían deberse al origen de donde son extraídas las algas.

Los polifenoles son un conjunto heterogéneo de moléculas que comparten la característica de poseer en su estructura varios grupos fenólicos y de tener potente actividad antioxidante para modular la actividad de diferentes enzimas, y en los mecanismos de señalización y procesos celulares $^{13}$. Los compuestos fenólicos son el grupo más extenso de sustancias no energéticas presentes en alimentos de origen vegetal y que están también presentes en las algas. El contenido de fenoles totales del alga roja Rhodymenia howeana fue de 980,2 \pm 0,07 mg EAG $/ 100 \mathrm{~g}$ de muestra seca; valores de estos metabolitos de $489 \mathrm{mg}$ EAG/100 g en Corallina officinalis evaluada por Ismail ${ }^{14}$ que representan cerca de la mitad del resultado del alga de estudio. Sin embargo, en Ceramium diaphanum, un alga roja, se encontró un valor de 1000 mg EAG/100 g según la investigación de Frikha et $a l^{3}$, lo que concuerda con nuestro estudio.

\section{CONCLUSIONES}

El alga marina roja Rhodymenia howeana es un alimento nutritivo por su alto valor proteico y bajo contenido calórico además de compuestos antioxidantes como polifenoles y niveles elevados de hierro y zinc, por lo que podría ser usado como suplemento de dietas para humanos y animales. 


\section{AGRADECIMIENTOS}

Se agradece enormemente a todos los docentes de la Maestría de Bioquímica de la Facultad de Medicina de la UNMSM por su labor académica y humana en el desarrollo de futuros investigadores, por su guía y paciencia para desarrollarse como maestrandos en este campo donde poseen vital experiencia.

\section{REFERENCIAS BIBLIOGRÁFICAS}

1. Acleto O. Algas Marinas del Perú de importancia económica. $2^{\mathrm{a}}$ ed. Lima: UNMSM Museo de Historia Natural; 1986.

2. Ortiz J. Composición nutricional y funcional de las algas rodofíceas chilenas [Internet]. Santiago, Chile: Universidad de Chile; 2011. [Citado 14 diciembre 2017]. Disponible en: http://www.repositorio.uchile.cl/handle/2250/121460

3. Frikha F, Kammoun M, Hammami N, Mchirgui R, Belbahri L, Gargouri L et al. Chemical composition and some biological activities of marine algae collected in Tunisia. Ciencias Marinas. 2011; 37(2): 113-124.

4. AOAC. Official Methods of Analysis of AOAC International (OMA). Gaithersburg, Maryland: AOAC International; 2005.

5. Jagota S, Dani H. A new colorimetric technique for estimation of vitamin c using Folin phenol reagent. Anal Biochem. 1982; 127 (1): 178-182.

6. Singleton V, Orthofer R, Lamuela R. Analysis of total phenols and other oxidation substrates and antioxidants by means of Folin-Ciocalteu reagent. Methods in enzymol. 1999; 299 (1): 152-178.

7. Xiren G, Aminah A. Proximate composition and total amino acid composition of Kappaphycus alvarezii found in the waters of Langkawi and Sabah, Malaysia. Int Food Res J. 2017; 24 (3): 1255-1260

8. Osman N, El-Manawy I, Amin A. Nutritional composition and mineral content of five macroalgae from red sea. Egytian J Phycol. 2011; 12(1): 89-102.

9. Álvarez R. Sierra L. Comparación bromatológica de las algas nativas (Gracilariopsis teunifrons, Sargassum filipéndula) y exóticas (Kappaphycus alvarezii) del Caribe Colombiano. Bol Cient Ms Hist Nat. 2009; 13(2): 17-25.

10. Hwang E, Ki K, Chung H. Proximate composition, amino acid, mineral, and heavy metal content of dried laver. Prev Nutr Food Sci. 2013; 18(2):139-144.

11. Ruíz M, Rubio C, Luis G, Ojeda I, González D, Gutiérrez A et al. Metales en algas comestibles: Evaluación y estudio comparativo. Rev Toxicol. 2013; 30 (1): 182-192.

12. Carbajal A. Manual de Nutrición y Dietética. [Internet] Universidad Complutense de Madrid; 2013. [citado 12 de mayo del 2018]. Disponible en: https://www.ucm.es/ nutricioncarbajal/

13. Criado C, Moya M. Vitaminas y antioxidante. [Internet] Madrid: Sanidad y ediciones SL; 2009. [citado 12 de mayo del 2018]. Disponible en: http://2011.elmedicointeractivo. com/Documentos/doc/VITAMINAS_Y_ANTIOX_EL_MEDICO.pdf 
14. Ismail G. Biochemical composition of some Egytian seaweeds with potent nutritive and antioxidant properties. Food Sci Technol. 2017; 37(2):294-302

15. Yildiz G, Vatan O, Celikler S, Dere S. Determination of the phenolic compounds and antioxidative capacity in red algae Gracilaria bursa-pastoris. Int J Food Prop. 2011; 14(3):496-502. 